\title{
Cytochrome c oxidase is activated by the oncoprotein Ras and is required for A549 lung adenocarcinoma growth
}

\author{
Sucheta Telang ${ }^{1}$, Kristin K Nelson', Deanna L Siow', Abdullah Yalcin', Joshua M Thornburg', \\ Yoannis Imbert-Fernandez ${ }^{1}$, Alden C Klarer ${ }^{1}$, Hanan Farghaly ${ }^{2}$, Brian F Clem ${ }^{1}$, John W Eaton ${ }^{1}$ and Jason Chesney ${ }^{{ }^{*}}$
}

\begin{abstract}
Background: Constitutive activation of Ras in immortalized bronchial epithelial cells increases electron transport chain activity, oxygen consumption and tricarboxylic acid cycling through unknown mechanisms. We hypothesized that members of the Ras family may stimulate respiration by enhancing the expression of the Vb regulatory subunit of cytochrome c oxidase (COX).

Results: We found that the introduction of activated $\mathrm{H}$-Ras ${ }^{\mathrm{V} 12}$ into immortalized human bronchial epithelial cells increased elF4E-dependent COX Vb protein expression simultaneously with an increase in COX activity and oxygen consumption. In support of the regulation of COX Vb expression by the Ras family, we also found that selective siRNA-mediated inhibition of K-Ras expression in A549 lung adenocarcinoma cells reduced COX Vb protein expression, COX activity, oxygen consumption and the steady-state concentration of ATP. We postulated that COX Vb-mediated activation of COX activity may be required for the anchorage-independent growth of A549 cells as soft agar colonies or as lung xenografts. We transfected the A549 cells with COX Vb small interfering or shRNA and observed a significant reduction of their COX activity, oxygen consumption, ATP and ability to grow in soft agar and as poorly differentiated tumors in athymic mice.
\end{abstract}

Conclusion: Taken together, our findings indicate that the activation of Ras increases COX activity and mitochondrial respiration in part via up-regulation of COX Vb and that this regulatory subunit of COX may have utility as a Ras effector target for the development of anti-neoplastic agents.

Keywords: Mitochondria, Glycolysis, Tricarboxylic acid cycle, Electron transport chain, Oncogene

\section{Background}

Activating mutations of the Ras guanosine nucleotidebinding proteins cause insensitivity to GTPase-activating proteins (GAP) and are common genetic alterations of human cancers [1]. Activated GTP-bound Ras family members promote survival and proliferation in part by increasing glucose uptake and flux into the synthesis of ribose-5-phosphate and lactate [2-4]. Recently, constitutive activation of Ras also has been found to increase mitochondrial tricarboxylic acid (TCA) cycle activity and oxygen consumption and to sensitize cells to the ATP-

\footnotetext{
* Correspondence: jason.chesney@louisville.edu

'Molecular Targets Program, James Graham Brown Cancer Center, University of Louisville, Louisville, KY, USA

Full list of author information is available at the end of the article
}

depleting and cytotoxic effects of electron transport perturbation using the complex I inhibitor rotenone [5]. These data suggested that activation of the Ras signalling pathway in immortalized cells might increase reliance on mitochondrial metabolism for energy and anabolism [6]. In support of this conclusion, disruption of mitochondrial function caused by loss of the mitochondrial transcription factor A (TFAM) gene reduced tumorigenesis in an oncogenic K-Ras-driven mouse model of lung cancer [7]. Additionally, elevated glycolytic flux into multiple mitochondrial TCA cycle products has been observed in human lung tumors in vivo [8] and these tumors commonly express mutated Ras [9]. Last, several agents that selectively disrupt mitochondrial function (termed mitocans) have been found to interfere with the

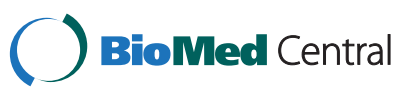


bioenergetic functions of cancer cells and suppress tumor growth $[10,11]$. Understanding the precise mechanisms whereby Ras family members regulate mitochondrial metabolism and the functional importance of this metabolic effect for the tumor-forming potential of Ras-transformed cells may yield new molecular targets for the development of anti-cancer agents.

The mitochondrial electron transport chain consists of four respiratory enzyme complexes that serve to create a proton gradient [12]. Cytochrome $c$ oxidase (COX), or complex IV, is the terminal enzyme in the electron transport chain which pumps protons from the matrix into the intermembrane space forming an electrochemical gradient across the inner mitochondrial membrane to generate ATP [13]. COX functions to catalyze electron transfer from cytochrome $c$ to molecular oxygen via four redox centers [14]. Although COX only has $20 \%$ total control over ATP synthesis [15], studies in vivo have indicated that COX represents the rate-limiting step of the electron transport chain [6]. COX is composed of thirteen different subunits $[16,17]$ - subunits I-III are encoded by the mitochondrial genome whereas the other ten subunits are encoded by nuclear genes [18]. The nuclear subunits are synthesized as precursor proteins and imported into the mitochondria where the complex is assembled [19]. Within the thirteen subunit complex, the nuclear-encoded subunit $\mathrm{Vb}$ serves a unique role in the regulation of COX activity as this particular subunit faces the matrix side and is sufficient to increase both COX activity and oxygen consumption in HeLa cervical adenocarcinoma cells [20]. Interestingly, the expression of subunit $\mathrm{Vb}$ has been found to be increased in neoplastic cells and to positively correlate with the progression of cells from normal epithelia to invasive carcinoma [21]. These observations suggest that COX Vb not only may serve as an essential regulatory subunit of COX but also may be mediating the increase in mitochondrial metabolism caused by activation of Ras [5].

In the current study, we demonstrate that introduction of the activated H-Ras ${ }^{\text {V12 }}$ GTPase into immortalized bronchial epithelial cells increases $\mathrm{COX} \mathrm{Vb}$ protein expression, $\mathrm{COX}$ activity and oxygen consumption and that $\mathrm{COX} \mathrm{Vb}$ protein expression is required to maintain the high oxygen consumption and ATP concentration of A549 lung adenocarcinoma cells that express oncogenic K-Ras. We also find that selective $\mathrm{COX} \mathrm{Vb}$ inhibition markedly inhibits
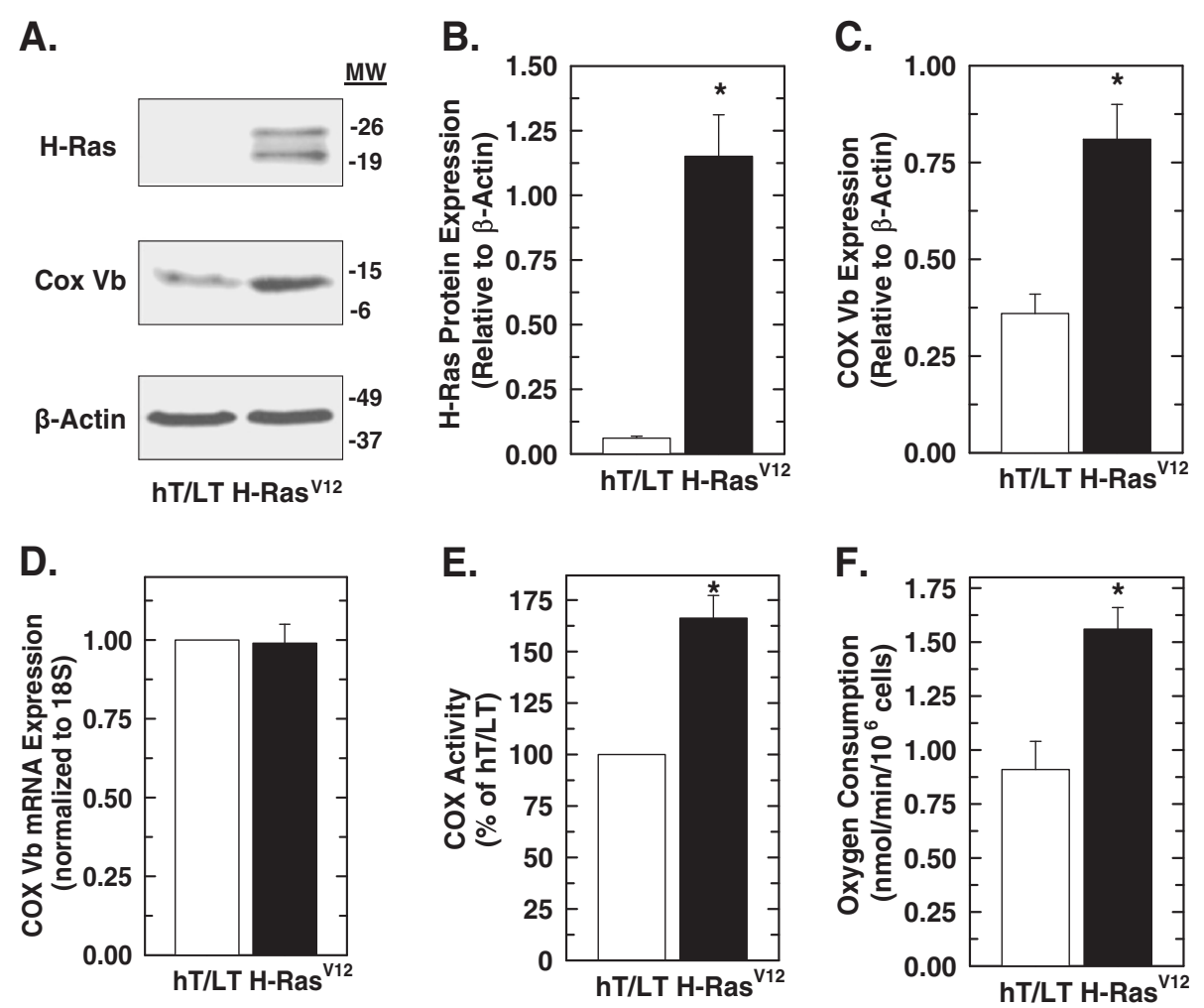

Figure 1 Introduction of oncogenic H-Ras ${ }^{\mathrm{V} 12}$ into immortalized human bronchial epithelial cells increases COX Vb protein expression, COX activity and oxygen consumption. hT/LT and H-Ras ${ }^{V / 2}$ cells were examined for H-Ras and COX Vb protein expression by Western blot analysis (A) and COX Vb mRNA expression by real-time RT-PCR (D). Densitometry of H-Ras (B) and COX Vb (C) protein expression relative to $\beta$-Actin expression was determined. Enzyme activity assays of $\operatorname{COX}(\mathbf{E})$ and oxygen consumption $(\mathbf{F})$ were performed as described. Data are expressed as the mean \pm SD of three experiments. ${ }^{*} p$ value $<0.05$ compared to hT/LT. 
soft agar colony formation and poorly differentiated A549 xenograft growth in mice without affecting proliferation in vitro. Taken together, these observations suggest that COX may be an appropriate molecular target for the development of anti-neoplastic agents.

\section{Results \\ $\mathrm{H}$-Ras ${ }^{\mathrm{V} 12}$ increases COX Vb protein expression and activity}

To explore the potential regulation of COX by Ras, we employed normal human bronchial epithelial cells that had been immortalized with the telomerase catalytic subunit and SV40 large T antigen (hT/LT cells) and then transformed with $\mathrm{H}-\mathrm{Ras}^{\mathrm{V} 12}\left(\mathrm{H}-\mathrm{Ras}^{\mathrm{V} 12}\right)$ [22]. Initially, we analyzed the hT/LT cells and H-Ras ${ }^{\mathrm{V} 12}$ cells for H-Ras protein expression by Western blot analysis and observed a marked increase in both wild-type H-Ras (lower band) and H-Ras ${ }^{\mathrm{V} 12}$ which displays reduced electrophoretic mobility [23] (upper band) (Figure 1A, B). These data suggest that introduction of $\mathrm{H}-\mathrm{Ras}^{\mathrm{V} 12}$ may activate a positive feedback loop that leads to increased wild-type $\mathrm{H}$-Ras expression. We then examined the cells for $\mathrm{COX} \mathrm{Vb}$ mRNA expression by real-time RT-PCR and for protein expression by Western blot and observed no increase in COX Vb mRNA (Figure 1D) but an increase in $\mathrm{COX} \mathrm{Vb}$ protein expression with the introduction of $\mathrm{H}-\mathrm{Ras}^{\mathrm{V} 12}$ (Figure 1A, C). The introduction of $\mathrm{H}-\mathrm{Ras}^{\mathrm{V} 12}$ into the hT/LT cells simultaneously increased COX activity (Figure 1E) and oxygen consumption (Figure 1F). Given that the only genetic difference between the immortalized hT/LT and H-Ras ${ }^{\mathrm{V} 12}$-transformed cells is the ectopic expression of $\mathrm{H}-\mathrm{Ras}^{\mathrm{V} 12}$, these results indicate that activation of Ras increases or stabilizes protein expression of $\mathrm{COX} \mathrm{Vb}$, stimulates $\mathrm{COX}$ activity and increases oxygen consumption.

\section{COX Vb protein expression is dependent on eukaryotic} translation initiation factor 4E (elF4E) expression Since H-Ras ${ }^{\mathrm{V} 12}$ expression had no effect on COX Vb mRNA but caused an increase in $\mathrm{COX} \mathrm{Vb}$ protein expression, we postulated that $\mathrm{H}-\mathrm{Ras}^{\mathrm{V} 12}$ may increase the efficiency of COX $\mathrm{Vb}$ mRNA translation. Activation of Ras signalling can increase the binding of the Eukaryotic Translation Initiation Factor 4E (eIF4E) to multiple mRNAs either through MAPK activation of Mnk which directly phosphorylates eIF4E or through the PI3K/ $\mathrm{AKT} / \mathrm{mTOR}$-mediated phosphorylation and inhibition of inhibitory $4 \mathrm{E}$ binding proteins [24]. Although an increase in eIF4E binding to the $5^{\prime}, 7$-methylguanosine cap structure of mRNAs stimulates the translation of all cap-dependent mRNAs, lengthy, GC-rich and highly structured 5' UTRs can markedly reduce efficient RNA unwinding by eIF4E. COX Vb has a long (266 bp), GCrich (56\%) 5'-UTR and we thus examined the consequences of selective siRNA-mediated eIF4E inhibition on the induction of $\mathrm{COX} \mathrm{Vb}$ protein expression caused by H-Ras $^{\mathrm{V} 12}$ (Figure 2A-C). Whereas silencing of eIF4E mRNA expression had no effect on $\mathrm{COX} \mathrm{Vb}$

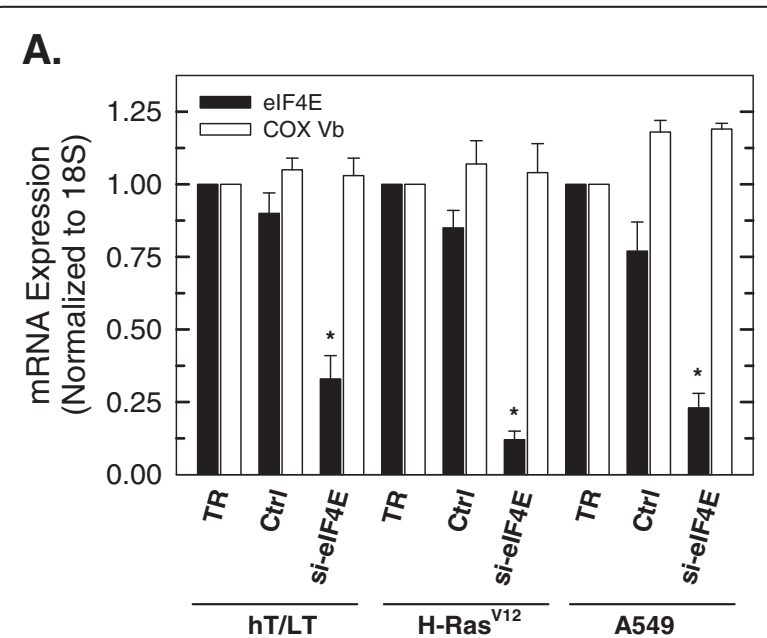

B.

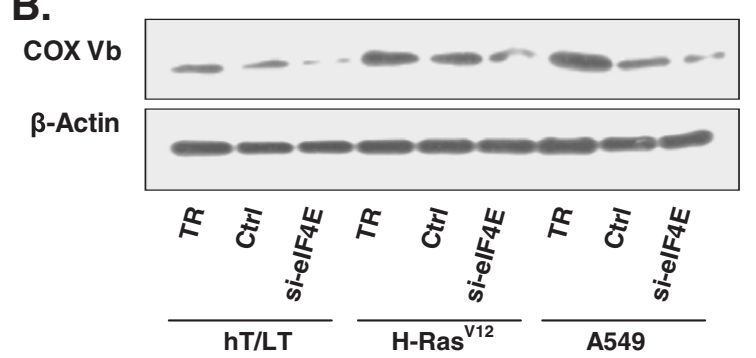

C.

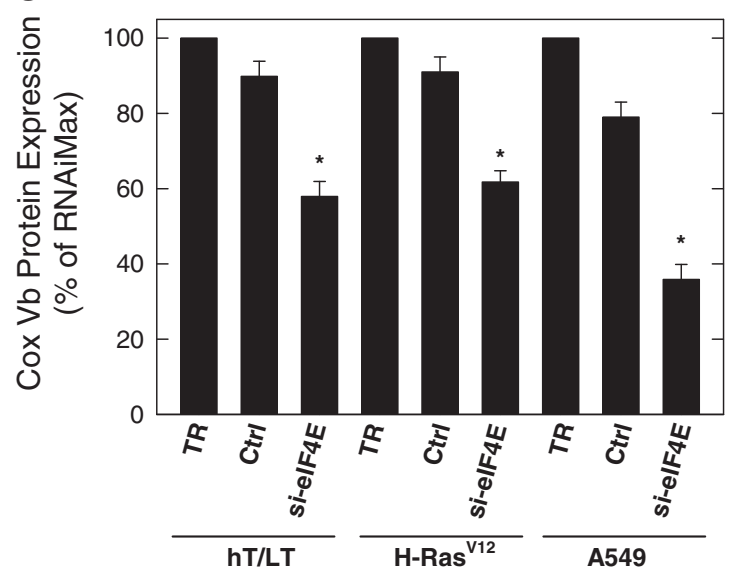

Figure 2 Selective inhibition of elF4E reduces COX $\mathrm{Vb}$ protein expression but has no effect on $\beta$-Actin protein expression. hT/LT, H-Ras ${ }^{\mathrm{V} 12}$ and A549 cells were transfected with transfection reagent alone, control siRNA or siRNA specific for elF4E for 48 hours and then examined for elF4E and COX Vb mRNA expression by real-time RT-PCR (A) and COX Vb and $\beta$-actin protein expression using Western blot analysis (B). Protein expression was quantified by densitometric analyses $(\mathbf{C})$ and expressed as percentage of transfection reagent. Data are expressed as the mean \pm SD of three experiments. ${ }^{*} p$ value $<0.05$. 
mRNA expression (Figure 2A), COX Vb protein expression in the hT/LT-immortalized, H-Ras ${ }^{\text {V12 }}$-transformed cells and $\mathrm{K}-\mathrm{Ras}^{\mathrm{S} 12}$ positive A549 lung adenocarcinoma cells was reduced by transfection with the eIF4E siRNA species (Figure 2B, C). Importantly, eIF4E siRNA transfection had no effect on $\beta$-actin protein expression, which contains a short non-complex 5'-UTR, in any of the three cell types examined (Figure 2B, C). These data indicate that $\mathrm{COX} \mathrm{Vb}$ protein expression is dependent on eIF4E activity and that the observed increase in COX $\mathrm{Vb}$ caused by introduction of oncogenic Ras can be partially attenuated by selective inhibition of eIF4E.

Selective inhibition of K-Ras suppresses COX Vb activity, oxygen consumption and intracellular ATP in A549 cells We next selectively inhibited the expression of K-Ras in A549 cells using siRNA and examined the consequences on $\mathrm{COX} \mathrm{Vb}$ protein expression, COX activity, oxygen consumption and ATP concentration. Transfection of A549 cells with K-Ras siRNA caused a reproducible reduction in $\mathrm{COX} \mathrm{Vb}$ protein expression (Figure 3A, B) without affecting COX Vb mRNA expression (mRNA fold change of $\mathrm{K}$-Ras siRNA relative to control siRNA = $1.01 \pm 0.12$ ) (Figure 3C).

Selective K-Ras inhibition also reduced COX activity, oxygen consumption and the steady-state concentration of ATP in the A549 cells (Figure 3D-F). These data coupled to the observations that ectopic expression of $\mathrm{H}$-Ras ${ }^{\mathrm{V} 12}$ increases COX Vb expression and COX activity further support the conclusion that $\mathrm{COX} \mathrm{Vb}$ expression is regulated by Ras signalling.

\section{Suppression of $\mathrm{COX} \mathrm{Vb}$ expression decreases anchorage-} independent growth as soft agar colonies

In order to directly examine the hypothesis that COX $\mathrm{Vb}$ expression is required for tumor growth, we next
A.

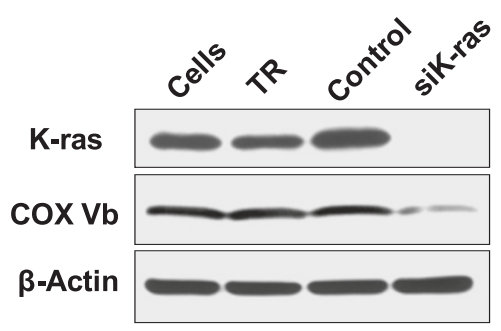

B.

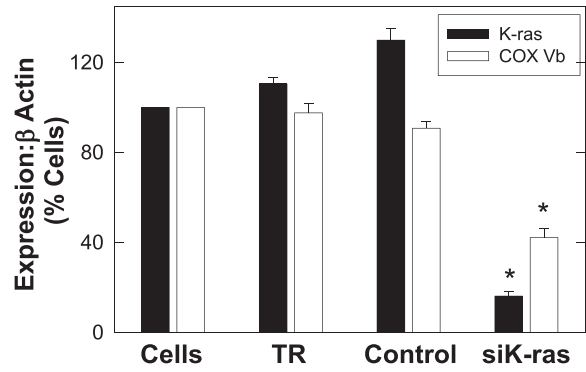

C.

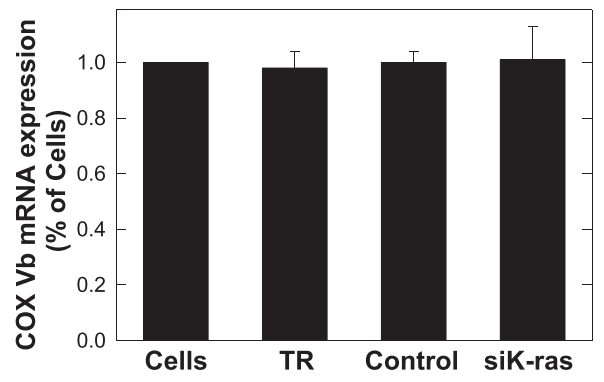

D.

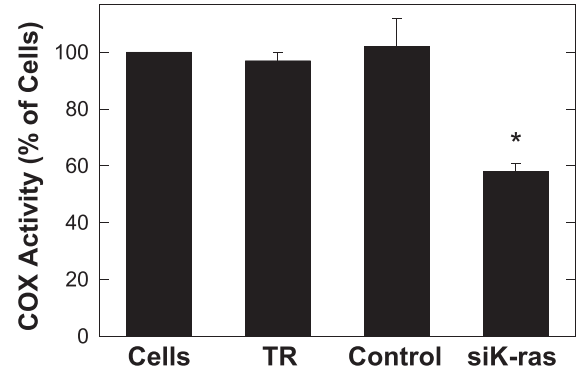

E.

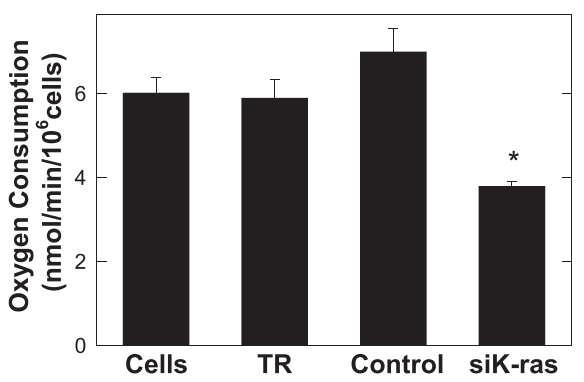

F.

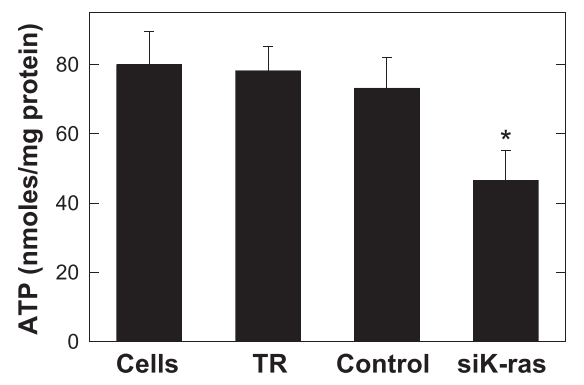

Figure 3 Selective inhibition of K-Ras expression by A549 lung adenocarcinoma cells reduces COX activity, oxygen consumption and ATP. A549 cells were transfected with transfection reagent (TR) alone, control siRNA or siRNA specific for K-Ras for 72 hours and then examined for COX Vb protein expression (A and B), COX Vb mRNA expression (C), COX activity (D), oxygen consumption (E) and ATP (F). Data are expressed as the mean \pm SD of three experiments. ${ }^{*} p$ value $<0.05$. 
transiently transfected the A549 cells with two COX Vb siRNA species specific for the open reading frame (Vb1) or for the 3'-untranslated region (Vb2). Forty eight hours after transfection, Western blot analyses indicated that both $\mathrm{COX} \mathrm{Vb}$ siRNA species inhibited the protein expression of $\mathrm{COX} \mathrm{Vb}$ (Figure 4A, B). We found that transfection of A549 cells with either COX Vb siRNA decreased COX activity, oxygen consumption, intracellular ATP and the NAD+/NADH ratio compared to controls (cells, transfection reagent alone or control siRNA) (Figures 4C-F). We next sought to assess the consequences of reduced mitochondrial metabolism on anchorage-independent survival and growth in soft agar. We observed no significant decrease in A549 anchoragedependent cell proliferation 48 hours after transfection with COX Vb-specific siRNA molecules (Figure 5A) but found that the siRNA-mediated silencing of $\mathrm{COX} \mathrm{Vb}$ markedly reduced subsequent A549 soft agar colony formation and growth (Figure 5B, C). Although the precise metabolic explanation for the apparent sensitivity of soft agar colony growth to $\mathrm{COX} \mathrm{Vb}$ inhibition is not well understood, nutrient and oxygen diffusion limitations imposed by adjacent cells within the colony combined with reduced COX activity may cause synergistic cytostasis.

\section{Suppression of $\mathrm{COX} \mathrm{Vb}$ expression decreases anchorage-} independent growth as tumors in athymic mice

The ability to grow as soft agar colonies can be predictive of tumor-forming capacity in athymic mice. We subcutaneously injected $5 \times 10^{6}$ live A549 cells stably transfected with a plasmid expressing the COX Vb1 shRNA or a control shRNA and measured tumor outgrowth using microcalipers. Suppression of $\mathrm{COX} \mathrm{Vb}$ protein expression in the COX Vb1 shRNA clone was confirmed by Western blot (Figure 6A). Although both
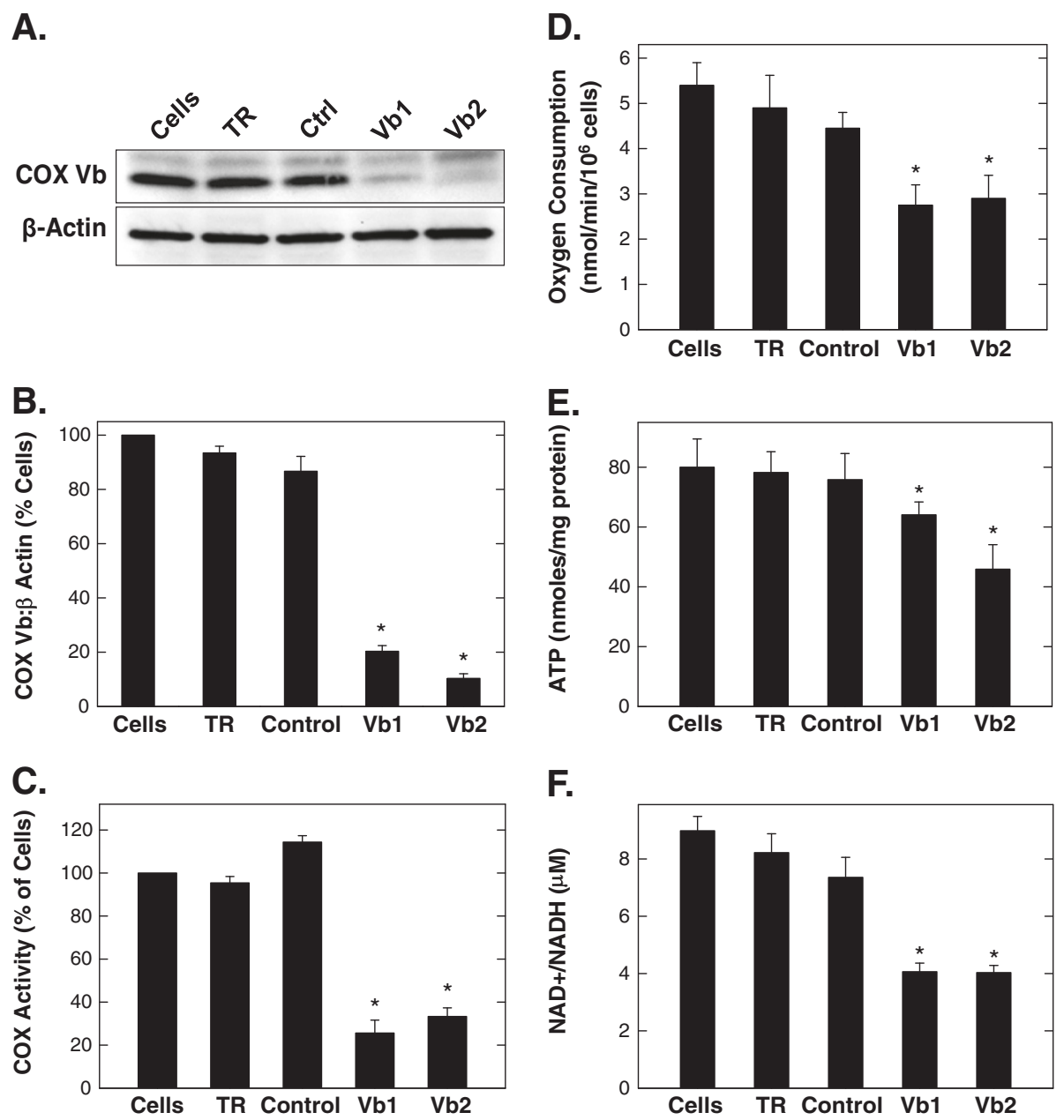

Figure 4 Selective inhibition of COX Vb by A549 cells reduces COX activity, oxygen consumption and ATP. A549 cells were transfected with transfection reagent alone, control siRNA or siRNA specific for the ORF (Vb1) or 3'-UTR (Vb2) of COX Vb for 48 hours and then examined for COX Vb protein expression $(\mathbf{A}$ and $\mathbf{B})$, COX activity $(\mathbf{C})$, oxygen consumption $(\mathbf{D})$, ATP $(\mathbf{E})$ and NAD+/NADH ratios $(\mathbf{F})$. Data are expressed as the mean \pm SD of three experiments. ${ }^{*} p$ value $<0.05$. 
A.

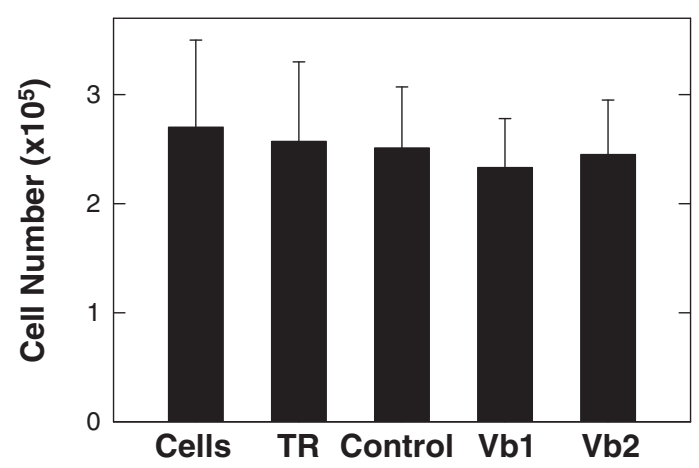

B.

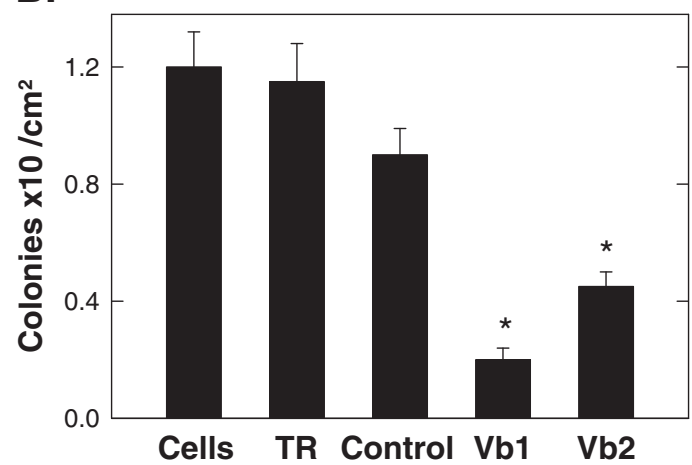

C.

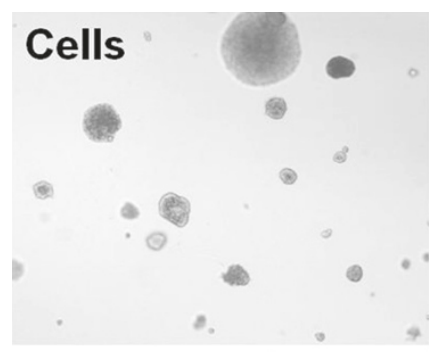

Control

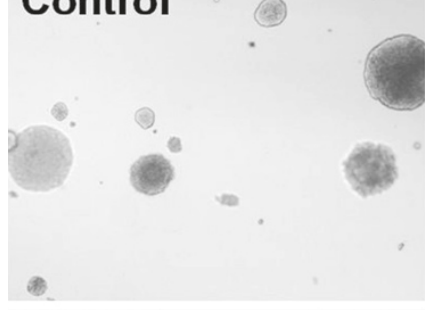

Vb1

Figure 5 Selective inhibition of COX Vb expression by A549 cells suppresses anchorage-independent growth in soft agar. A. $10^{5}$ A549 cells transfected with COX Vb or control siRNA were cultured for 48 hours and viable cells were enumerated using light microscopy. B. After the 48 hour transfection, A549 cells were plated on $6 \mathrm{~cm}$ dishes containing DMEM with $0.6 \%$ agar. Cells were fed with $0.2 \%$ agar in media every three days. After 21 days, soft agar colonies were enumerated. C. Representative photomicrographs of the soft agar colonies. Data are expressed as the mean \pm SD of five (A) and three (B) experiments. ${ }^{*} p$ value $<0.05$.

groups of mice had detectable subcutaneous A549 tumors after 4 weeks, the outgrowth of the COX Vb1 shRNA expressing tumors was markedly reduced over the following 3 weeks (Figure 6B). We then euthanized the mice, excised the tumors and stained them with Hematoxylin and Eosin (Figure 6C-H). We found that the control A549 tumors contained large areas of tumor necrosis (see white arrows, Figure 6D), pleomorphic nuclei (black arrows, Figure 6E) and numerous mitotic figures (white arrows, Figure 6E), all of which are consistent with high-grade, rapidly growing but poorly differentiated adenocarcinomas. In contrast, we observed no tumor necrosis, low mitotic activity, and few pleomorphic nuclei in the COX Vb1 shRNA-transfected A549 tumors (Figure 6F-H), indicating that $\mathrm{COX} \mathrm{Vb}$ inhibition in the A549 cells significantly lowered the histopathological grade of tumors in vivo. Given that COX $\mathrm{Vb}$ inhibition reduces oxygen consumption in vitro, we speculated that the oxygen concentration may be increased in the COX Vb shRNA A549 tumors relative to the control tumors. However, we examined the tumors for carbonic anhydrase IX protein expression, which is a target gene of HIF- $1 \alpha$, and observed no difference in expression (data not shown).

\section{Discussion}

In this study, we found that introduction of oncogenic $\mathrm{H}-\mathrm{Ras}^{\mathrm{V} 12}$ into immortalized human bronchial epithelial cells enhanced eIF4E-dependent $\mathrm{COX} \mathrm{Vb}$ protein expression, COX activity and oxygen consumption. Conversely, selective siRNA silencing of K-Ras expression in A549 cells was observed to selectively suppress COX Vb protein expression, COX activity and oxygen consumption. Transfection of the A549 cells with two independent siRNA molecules specific for $\mathrm{COX} \mathrm{Vb}$ caused a marked decrease in COX activity, oxygen consumption, ATP, anchorage-independent soft agar colony growth and xenograft growth in athymic mice. Taken together, these data suggest that a metabolic regulatory pathway thus exists whereby Ras signalling activates eIF4E activity which, in turn, leads to increased $\mathrm{COX} \mathrm{Vb}$ protein expression, COX activity and oxygen consumption. The 
A.

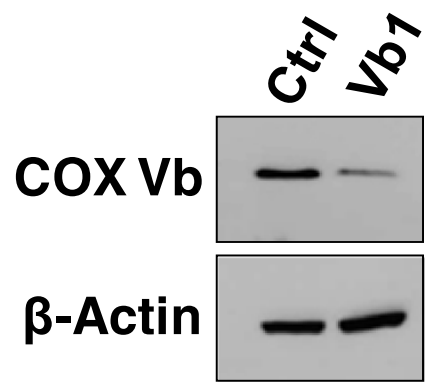

B.

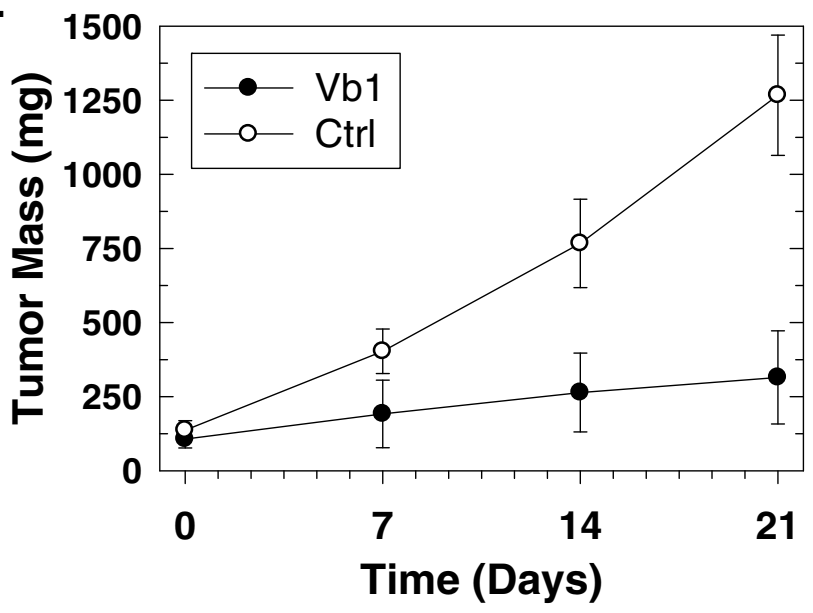

C.
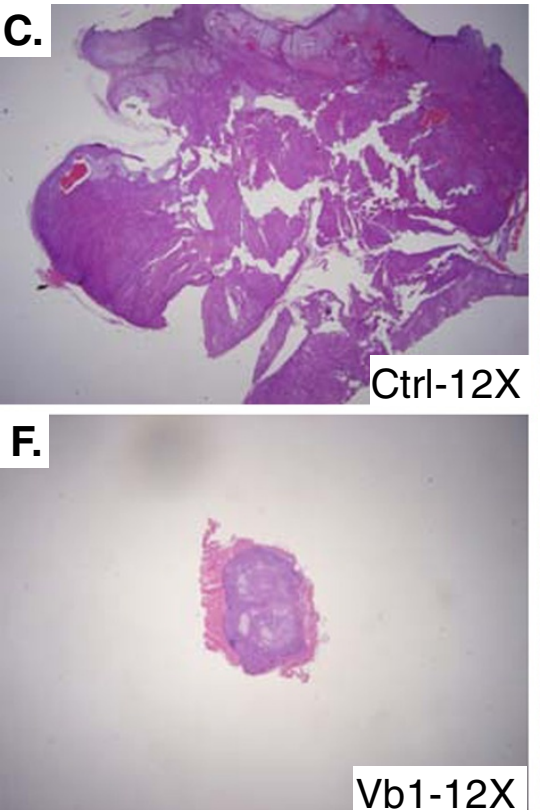
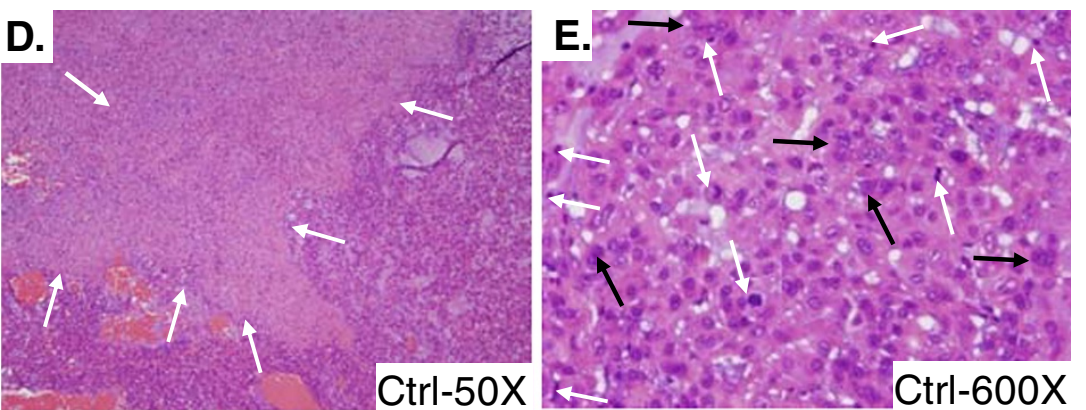

G.

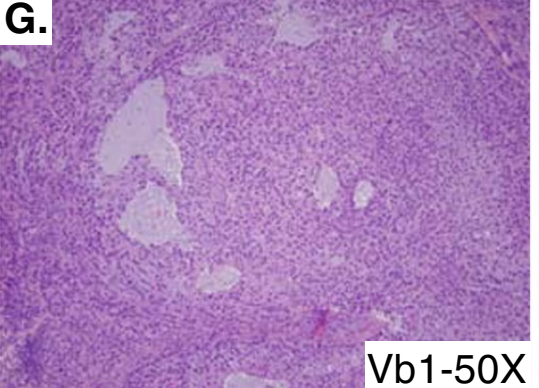

$\mathrm{H}$

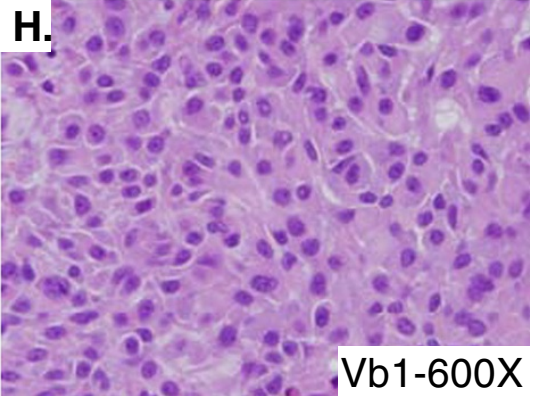

Figure 6 Stable COX Vb shRNA expression in A549 cells reduces the outgrowth of A549 xenografts and inhibits multiple histopathological indicators of poor differentiation in athymic mice. $5 \times 10^{6} \mathrm{~A} 549$ cells stably transfected with control or COX Vb-specific shRNA were subjected to Western blot analysis for COX Vb or $\beta$-actin expression (A) and then injected subcutaneously into athymic mice. After 4 weeks, tumors were measured weekly for a total of 3 additional weeks (timepoints $0,7,14$ and 21) using microcalipers. Tumor mass was calculated based on bidimensional measurements and data are expressed as the mean \pm SEM of two experiments (B). After 7 weeks of growth, mice were euthanized and tumors were excised, fixed in formalin, paraffin-embedded, sectioned and stained with hematoxylin/eosin. Light micrographs demonstrate that, relative to the COX Vb1 shRNA-transfected A549 tumors (F-H), the control A549 tumors were higher grade, poorly differentiated and invasive (C-E), contain large areas of tumor necrosis (D; white arrows), pleomorphic nuclei (E, black arrows), and numerous mitotic figures (E, white arrows).

reduction in xenograft growth that was caused by COX $\mathrm{Vb}$ inhibition was associated with reduced histological markers of high-grade poorly differentiated cells which may indicate that COX activity is required for the aggressiveness of certain lung adenocarcinomas. These observations support an examination of potential correlations between $\mathrm{COX} \mathrm{Vb}$ protein expression by human lung adenocarcinomas and clinical outcomes including growth rates, response to therapy and overall survival. Additionally, these results indicate that COX may have utility as a target for the development of anti-cancer therapeutics, especially against tumors in which the Ras signalling pathway is activated.

Ras proto-oncogenes encode membrane-bound $21 \mathrm{kDa}$ guanosine nucleotide-binding proteins that transduce mitogenic signals from tyrosine-kinase 
receptors. Whereas Ras mutations occur in $\sim 30$ percent of human cancers [9], activation of Ras signalling pathways (e.g. via epidermal growth factor receptor [EGFR] amplifications) is a nearly ubiquitous characteristic of human cancers. K-Ras ${ }^{G 12 D}$ transgenic mice develop tumors which express $\mathrm{COX} \mathrm{Vb}$ [25], and activation of the Ras/cAMP/protein kinase A pathway in Saccharomyces cerevisiae increases COX activity and oxygen consumption [26]. Additionally, the ratio of $\mathrm{COX} \mathrm{Vb}$ to COX I or COX II expression markedly increases during the progression from normal human prostate epithelium to invasive prostate carcinoma [21], a transformation that is dependent on the downstream Ras signalling pathways MAPK and PI3K/AKT [27]. The current study adds to this body of literature, demonstrating that the discrete introduction of the human oncogene $\mathrm{H}-\mathrm{Ras}^{\mathrm{V} 12}$ into immortalized human bronchial epithelial cells not only increases protein expression of the regulatory $\mathrm{Vb}$ subunit but also oxygen consumption and COX activity. Perhaps more importantly, the current study demonstrates for the first time that $\mathrm{COX} \mathrm{Vb}$ expression by Ras-transformed lung adenocarcinoma cells is required for their ability to grow as a high-grade, poorly differentiated tumor in athymic mice.

We previously reported that introduction of activated Ras into immortalized cells simultaneously increased glycolytic flux to lactate, TCA cycle activity and oxygen consumption [5]. Ras oncoproteins also directly increase the expression of uncoupling protein (UCP)-1, and UCP-2 expression has been found to be increased in most human colon adenocarcinomas in which up to $60 \%$ contain K-Ras mutations $[28,29]$. The mitochondrial TCA cycle and cytoplasmic GADPH reactions require oxidized NAD+ which is produced by $\mathrm{LDH}$ in the cytosol or by electron transport chain activity in the mitochondria. We postulate that Ras activation may cause simultaneous stimulation of COX activity and uncoupling of ATP synthase from the proton gradient in order to facilitate the continued oxidation of NADH to NAD+ necessary for glycolytic flux and TCA cycling [6].

Expression of oncogenic H-Ras ${ }^{\mathrm{V} 12}$ sensitizes immortalized cells to electron transport chain disruption with rotenone suggesting that activation of Ras signalling also may confer greater reliance on the electron transport chain for energy requirements [5]. Given that we have found that introduction of $\mathrm{H}-\mathrm{Ras}^{\mathrm{V} 12}$ increases $\mathrm{COX} \mathrm{Vb}$ protein expression and COX activity relative to immortalized cells, we speculate that the $\mathrm{COX} \mathrm{Vb}$ subunit and the entire COX complex serve as essential metabolic downstream effectors of Ras. Dichloroacetate (DCA), an inhibitor of pyruvate dehydrogenase (PDH) kinase and thus a stimulator of PDH and flux into the TCA cycle, has been observed to increase oxygen consumption but to reduce tumor growth [30]. In contrast, we have found that selective inhibition of $\mathrm{COX} \mathrm{Vb}$ reduced both oxygen consumption and tumor growth. Although the precise mechanism and rationale for these differences are not well understood, we postulate that the disruption of NADH oxidation caused by either the diversion of pyruvate away from $\mathrm{LDH}$ or by the inhibition of electron transport chain activity (Figure 4F) may yield a similarly deleterious effect on anabolic pathways such as the TCA cycle that are required for tumor growth.

\section{Conclusion}

In conclusion, we find that activation of the Ras signalling pathway increases COX activity in part via eIF4Edependent enhancement of $\mathrm{COX} \mathrm{Vb}$ protein expression and that COX is required for tumorigenic growth. The continued characterization of the regulatory mechanisms and rationale for increased COX activity in transformed cells should allow us to tailor rational anti-metabolic approaches for the treatment of cancer.

\section{Methods}

\section{Cell lines and culture}

A549 human lung adenocarcinoma cells (American Type Culture Collection, Manassas, VA) were maintained in Dulbecco's Modified Eagle Medium (DMEM, Invitrogen, Grand Island, NY) supplemented with 10\% FCS and gentamicin sulfate (HyClone, Logan, UT). Normal human bronchial epithelial cells expressing telomerase and SV40 large $\mathrm{T}$ antigen (hT/LT) and activated Ras $\left(\mathrm{H}-\mathrm{Ras}^{\mathrm{V} 12}\right)$ were gifts from Dr. B. J. Rollins, Dana Farber Cancer Institute. hT/LT and H-Ras ${ }^{\mathrm{V} 12}$ cells were grown in BEGM medium formulated with SingleQuots (bovine pituitary extract, recombinant human epidermal growth factor, hydrocortisone, insulin, epinephrine, tri-iodothyronine, transferrin, gentamicin, amphotericin B and retinoic acid) (Lonza, Walkersville, MD). Media contained physiologic glucose $(5.5 \mathrm{mM})$ and glutamine $(1 \mathrm{mM})$, and cells were cultured under $21 \% \mathrm{O}_{2}$ (normoxia) with $5 \% \mathrm{CO}_{2}$ at $37^{\circ} \mathrm{C}$ (Heraeus).

\section{siRNA transfections}

Cells were plated at $8 \times 10^{4}$ cells/well in 6 well tissue culture plates and incubated at $5 \% \mathrm{CO}_{2}$ and $37^{\circ} \mathrm{C}$ in DMEM as described above for 24 hours. Cells were then transfected with siRNA using RNAiMax (Invitrogen, Carlsbad, CA) according to the manufacturer's protocol. For inhibition of COX Vb in A549 cells, two siRNA constructs were designed, one specific for the open reading frame region of $\mathrm{COX} \mathrm{Vb}(\mathrm{COX} \mathrm{Vb}-\mathrm{ORF})$ and a second specific for the 3' untranslated region (COX Vb3'UTR), termed $\mathrm{COX} \mathrm{Vb} 1$ and $\mathrm{COX} \mathrm{Vb} 2$ respectively. The target sequences (sense strands) were as follows: $\mathrm{Vb} 1=$ AGUAGGCUGCAUCUGUGAA and $\mathrm{Vb} 2=$ CAGUAAAGACUAGCCAUUG. siRNAs against lamin 
$\mathrm{A} / \mathrm{C}$ and RNAiMax alone were included as controls. $\mathrm{COX} \mathrm{Vb}$ siRNA duplexes were synthesized by Dharmacon (Lafayette, CO). For the inhibition of eIF4E, hT/LT, $\mathrm{H}-\operatorname{Ras}^{\mathrm{V} 12}$ or A549 cells were transfected with presynthesized eIF4E siRNA (Ambion, Austin, TX). For the inhibition of K-Ras, A549 cells were transfected with pooled K-Ras siRNA (Santa Cruz Biotechnology, Santa Cruz, CA).

\section{Western blot analysis}

Western blot analysis was carried out on protein lysates from A549, hT/LT, and H-Ras ${ }^{\mathrm{V} 12}$ cells transfected with siRNA as described above. Briefly, equal amounts of total protein $(30 \mu \mathrm{g})$ from each sample were run on a 4$20 \%$ gradient SDS-PAGE gel under reducing conditions and proteins were transferred to PVDF membranes (BioRad, Hercules, CA). Membranes were probed with a 1:500 dilution of anti-COX $\mathrm{Vb}$ polyclonal mouse antibody (Invitrogen), a 1:1000 dilution of anti-H-Ras antibody or a 1:100 dilution of anti-K-Ras antibody (both from Santa Cruz). Immunoreactive proteins were visualized by incubating membranes with horseradish peroxidase-conjugated anti-mouse or anti-rabbit IgG antibody (1:10000) followed by reaction with ECL Plus (Amersham, St. Louis MO). Membranes were subsequently probed with a mouse monoclonal $\beta$-actin antibody (Sigma, St. Louis MO) as an internal protein loading control. Experiments were repeated four times. All data are expressed as the mean \pm SD of three experiments. Statistical significance was assessed by the unpaired two-tail $T$-test.

\section{COX activity assay}

COX activity was measured as described in Campian et al. [20]. Briefly, lysates of cells (at a concentration of $2 \times 10^{6}$ cells $/ \mathrm{ml}$ ) were prepared in $0.25 \mathrm{M}$ sucrose, $40 \mathrm{mM}$ potassium chloride, $2 \mathrm{mM}$ EGTA, $1 \mathrm{mg} / \mathrm{ml}$ bovine serum albumin, and $20 \mathrm{mM}$ Tris- $\mathrm{HCl}(\mathrm{pH} 7.2)$ and disrupted by 31 second bursts using a microtip Fisher model 100 sonic dismembrator at scale 3 (on a scale of 0-10 of the 100-watt maximum power output) (Fisher Scientific, Pittsburgh, PA). The lysate was centrifuged at $4000 \times g$ for $10 \mathrm{~min}$, the pellet discarded and the supernatant was used for COX assays. Assays contained $30 \mu \mathrm{g}$ of protein and were performed at $37^{\circ} \mathrm{C}$ in $200 \mu \mathrm{l}$ reaction volumes. The assay involved the addition of $40 \mu \mathrm{M}$ ferrocytochrome $c$ in an isosmotic medium $(10 \mathrm{mM}$ $\mathrm{KH}_{2} \mathrm{PO}_{4}$ (pH 6.5), $1 \mathrm{mg} / \mathrm{ml}$ bovine serum albumin, $0.3 \mathrm{M}$ sucrose) containing $2.5 \mathrm{mM} n$-dodecyl maltoside to permeabilize mitochondrial membranes. The activity was calculated from the rate of decrease in absorbance of ferrocytochrome $c$ at $550 \mathrm{~nm}\left(\varepsilon=19.1 \mathrm{mM}^{-1} \mathrm{~cm}^{-1}\right)$. All data are expressed as the mean $\pm \mathrm{SD}$ of three experiments. Statistical significance was assessed by the unpaired two-tail $T$-test.

\section{Oxygen consumption}

Cells were detached with trypsin-EDTA and centrifuged at $1000 \mathrm{rpm}$ for 5 minutes in complete culture medium. Oxygen consumption was measured using a Strathkelvin 782 Oxygen Meter (Strathkelvin Instruments, Glasgow, Scotland, UK). Respiration rates were measured using $1 \times$ $10^{6}$ cells suspended in a total volume of $525 \mu \mathrm{L}$ DMEM containing $10 \% \mathrm{FBS}$ at $37^{\circ} \mathrm{C}$ for 10 minutes. A starting $\mathrm{O}_{2}$ concentration of $240 \mu \mathrm{M}$ was assumed based on $\mathrm{O}_{2}$ solubility at conditions in this laboratory $\left(1 \mathrm{~atm} / 37^{\circ} \mathrm{C}\right)$. Experiments were repeated four times. The data shown are mean \pm SD of three experiments. Statistical significance was assessed by the unpaired two-tail $T$-test.

\section{ATP assay}

Cells were washed (while still adherent) with cold PBS $\mathrm{x} 1$, lysed with passive lysis buffer (1X, Molecular Probes, Invitrogen) added directly to the plates and immediately harvested by scraping. The lysates were flash frozen (to $-80^{\circ} \mathrm{C}$ ) and thawed (to $37^{\circ} \mathrm{C}$ ) once to accomplish complete lysis and then centrifuged $\left(\right.$ at $\left.4^{\circ} \mathrm{C}\right)$ for $30 \mathrm{sec}-$ onds to clear the lysates. Intracellular ATP levels were determined using a bioluminescence assay (Molecular Probes) utilizing recombinant firefly luciferase and its substrate, D-luciferin. The luminescence was read in a TD-20/20 luminometer (Turner Designs, Sunnyvale, CA) at $560 \mathrm{~nm}$. The ATP values were calculated using an ATP standard curve. The protein concentrations of the lysates were estimated using the bicinchoninic acid (BCA) assay (Pierce Biotechnology, Rockford, IL) and ATP was expressed as nmol per mg protein. Experiments were repeated five times. All data are expressed as the mean \pm s.d. of three experiments. Statistical significance was assessed by the unpaired two-tail $T$-test.

\section{$\mathrm{NAD+} / \mathrm{NADH}$ ratio assay}

A549 cells treated with siRNA species for 48 hours were lifted using trypsin - EDTA, washed twice with cold PBS and $10^{5}$ cells were pelleted by centrifugation. NAD+ and NADH concentrations were measured using an enzymebased assay (E2ND-100, Bioassay Systems, Hayward, CA). Briefly, the cell pellets were homogenized in either $\mathrm{NAD}+$ extraction buffer (containing $0.40 \% \mathrm{HCl}$ ) or $\mathrm{NADH}$ extraction buffer (containing $0.40 \% \mathrm{NaOH}$ ), extracts heated to $60^{\circ} \mathrm{C}$ and then assay buffer (containing Tris(hydroxymethyl)aminomethane $3.0 \%$, and BSA $0.10 \%$ ) and extraction buffer (to neutralize the extracts) were added. Following centrifugation, supernatants were mixed with working reagent containing assay buffer, lactate dehydrogenase, diaphorase, lactate and tetrazolium dye (MTT) and optical density at $565 \mathrm{~nm}$ was recorded 
at time zero and at 15 minutes using a 96 well plate reader spectrophotometer. The difference in absorbance was compared with standard solutions and used to calculate NADH and NAD+ concentrations. All data are expressed as the mean \pm s.d. of three experiments. Statistical significance was assessed by the unpaired two-tail T-test.

\section{Cell viability}

For measurements of contact-dependent growth, cells were grown in appropriate media as described above. Following siRNA transfections, cells were grown for $48 \mathrm{~h}$. Proliferation was determined using trypan blue exclusion. Cells were incubated in 20\% trypan blue (Sigma) for 5 minutes. Cells excluding trypan blue were counted using a standard hemocytometer (Hausser Scientific) to determine the total number of viable cells. All data are expressed as the mean \pm SD of five experiments. Statistical significance was assessed by the unpaired two-tail $T$-test.

\section{Soft agar colony formation assay}

A549 cells with or without $\mathrm{Vb}$ siRNA transfection for 48 hours were plated at a density of $1 \times 10^{4}$ cells $/ 60-\mathrm{mm}$ plate sandwiched by $3 \mathrm{ml}$ bottom agar $(0.6 \%)$ and $3 \mathrm{ml}$ top agar $(0.3 \%)$. Cells were fed every three days by adding a new layer of top agar. After 21 days, colonies were counted in random $1 \mathrm{~cm}^{2}$ sections of each plate. All data are expressed as the mean $\pm S D$ of three experiments. Statistical significance was assessed by the unpaired twotail $T$-test.

\section{Xenograft studies}

A549 lung adenocarcinoma cells stably transfected with either $\mathrm{COX} \mathrm{Vb}$ or a control shRNA were collected from exponential growth phase culture in DMEM supplemented with $10 \%$ FCS. Cells were washed twice and re-suspended in PBS $\left(5 \times 10^{7}\right.$ cells $\left./ \mathrm{ml}\right)$. Groups of CD1 female athymic mice $(20 \mathrm{gm})$ were injected s.c. with $0.1 \mathrm{ml}$ of the cell suspension $\left(5 \times 10^{6}\right.$ cells $)$. The tumors were followed from the time of appearance until 21 days. Tumor masses were determined in a blinded fashion with Vernier calipers according to the following formula: $\operatorname{mass}(\mathrm{mg})=(\text { width }, \mathrm{mm})^{2} \times($ length, $\mathrm{mm}) / 2$ [31]. All data are expressed as the mean $\pm \mathrm{SD}$ of two experiments ( $\mathrm{n}=8$ per group). Statistical significance was assessed by the unpaired two tail $T$-test. At the end of the experiment, mice were euthanized and the tumors were excised, fixed in formalin, embedded in paraffin and stained with Hematoxylin and Eosin or with anti-CA IX (Abcam, Cambridge, MA).

\section{Competing interests}

The authors declare that they have no competing interests.

\section{Authors' contributions}

ST conducted the K-ras and COX Vb siRNA experiments, soft agar and in vivo experiments and participated in the conception of the manuscript. KKN assisted with the COX assay. DLS conducted the EIF4E siRNA experiments. AY assisted in the in vivo experiments. JMT assisted in the development of the COX assay. YI assisted in the in vivo experiments. ACK conducted the real time RT-PCR. HF conducted the histopathological review of the tumors. BFC assisted with the oxygen consumption experiments. JWE participated in the design of the experiments and interpretation of the data. JC conceived the research, directed all experiments and drafted the manuscript. All the authors have been involved in the drafting of the manuscript and have read and approved the final manuscript

\section{Acknowledgements}

This work was supported by the Kentucky Lung Cancer Research Program (JC), the James Graham Brown Cancer Center (JC), the National Cancer Institute (1 R01 CA116428-01; JC, 1 R01 CA140991-01; ST) and the American Cancer Society (RSG-10-021-01-CNE; ST). We gratefully acknowledge helpful discussions with Otto Grubraw.

\section{Author details}

${ }^{1}$ Molecular Targets Program, James Graham Brown Cancer Center, University of Louisville, Louisville, KY, USA. ²Department of Pathology, University of Louisville, Louisville, KY, USA

Received: 25 May 2012 Accepted: 17 August 2012

Published: 23 August 2012

\section{References}

1. Roberts PJ, Der CJ: Targeting the Raf-MEK-ERK mitogen-activated protein kinase cascade for the treatment of cancer. Oncogene 2007, 26:3291-3310

2. Kole HK, Resnick RJ, Van Doren M, Racker E: Regulation of 6-phosphofructo-1-kinase activity in ras-transformed rat-1 fibroblasts. Arch Biochem Biophys 1991, 286:586-590.

3. Racker E, Resnick RJ, Feldman R: Glycolysis and methylaminoisobutyrate uptake in rat-1 cells transfected with ras or myc oncogenes. Proc Natl Acad Sci U S A 1985, 82:3535-3538.

4. Ramanathan A, Wang C, Schreiber SL: Perturbational profiling of a cell-line model of tumorigenesis by using metabolic measurements. Proc Natl Acad Sci U S A 2005, 102:5992-5997.

5. Telang S, Lane AN, Nelson KK, Arumugam S, Chesney J: The oncoprotein H-RasV12 increases mitochondrial metabolism. Mol Cancer 2007, 6:77.

6. Chesney J, Telang S: Regulation of Glycolytic and Mitochondrial Metabolism by Ras. Curr Pharm Biotechnol 2012, [Epub ahead of print].

7. Weinberg F, Hamanaka R, Wheaton WW, Weinberg S, Joseph J, Lopez M, Kalyanaraman B, Mutlu GM, Budinger GR, Chandel NS: Mitochondrial metabolism and ROS generation are essential for Kras-mediated tumorigenicity. Proc Natl Acad Sci U S A 2010, 107:8788-8793.

8. Fan TW, Lane AN, Higashi RM, Farag MA, Gao H, Bousamra M, Miller DM: Altered regulation of metabolic pathways in human lung cancer discerned by (13)C stable isotope-resolved metabolomics (SIRM). Mol Cancer 2009, 8:41.

9. Minamoto T, Mai M, Ronai Z: K-ras mutation: early detection in molecular diagnosis and risk assessment of colorectal, pancreas, and lung cancers-a review. Cancer Detect Prev 2000, 24:1-12.

10. Dong LF, Freeman R, Liu J, Zobalova R, Marin-Hernandez A, Stantic M, Rohlena J, Valis K, Rodriguez-Enriquez S, Butcher B, et al: Suppression of tumor growth in vivo by the mitocan alpha-tocopheryl succinate requires respiratory complex II. Clin Cancer Res 2009, 15:1593-1600.

11. Ralph SJ, Rodriguez-Enriquez S, Neuzil J, Moreno-Sanchez R: Bioenergetic pathways in tumor mitochondria as targets for cancer therapy and the importance of the ROS-induced apoptotic trigger. Mol Aspects Med 2010, 31:29-59.

12. Gibson BW: The human mitochondrial proteome: oxidative stress, protein modifications and oxidative phosphorylation. Int J Biochem Cell Biol 2005, 37:927-934

13. Kadenbach B, Huttemann M, Arnold S, Lee I, Bender E: Mitochondrial energy metabolism is regulated via nuclear-coded subunits of cytochrome c oxidase. Free Radic Biol Med 2000, 29:211-221. 
14. Kadenbach B, Merle P: On the function of multiple subunits of cytochrome coxidase from higher eukaryotes. FEBS Lett 1981, 135:1-11.

15. Brookes PS, Levonen AL, Shiva S, Sarti P, Darley-Usmar VM: Mitochondria: regulators of signal transduction by reactive oxygen and nitrogen species. Free Radic Biol Med 2002, 33:755-764.

16. Capaldi RA: Structure and function of cytochrome c oxidase. Annu Rev Biochem 1990, 59:569-596.

17. Kadenbach B, Jarausch J, Hartmann R, Merle P: Separation of mammalian cytochrome $\mathrm{c}$ oxidase into 13 polypeptides by a sodium dodecyl sulfate-gel electrophoretic procedure. Anal Biochem 1983, 129:517-521.

18. Anderson WF, Ohlendorf DH, Takeda Y, Matthews BW: Structure of the cro repressor from bacteriophage lambda and its interaction with DNA. Nature 1981, 290:754-758.

19. Capaldi RA, Marusich MF, Taanman JW: Mammalian cytochrome-c oxidase: characterization of enzyme and immunological detection of subunits in tissue extracts and whole cells. Methods Enzymol 1995, 260:117-132.

20. Campian JL, Gao X, Qian M, Eaton JW: Cytochrome C oxidase activity and oxygen tolerance. J Biol Chem 2007, 282:12430-12438.

21. Herrmann PC, Gillespie JW, Charboneau L, Bichsel VE, Paweletz CP, Calvert VS, Kohn EC, Emmert-Buck MR, Liotta LA, Petricoin EF 3rd: Mitochondrial proteome: altered cytochrome $\mathrm{c}$ oxidase subunit levels in prostate cancer Proteomics 2003, 3:1801-1810.

22. Soejima K, Fang W, Rollins BJ: DNA methyltransferase $3 \mathrm{~b}$ contributes to oncogenic transformation induced by SV40T antigen and activated Ras. Oncogene 2003, 22:4723-4733.

23. Srivastava SK, Yuasa Y, Reynolds SH, Aaronson SA: Effects of two major activating lesions on the structure and conformation of human ras oncogene products. Proc Natl Acad Sci U S A 1985, 82:38-42.

24. Mamane Y, Petroulakis E, Rong L, Yoshida K, Ler LW, Sonenberg N elF4E-from translation to transformation. Oncogene 2004, 23:3172-3179.

25. Lee S, Kang J, Cho M, Seo E, Choi H, Kim E, Kim J, Kim H, Kang GY, Kim KP, et al: Profiling of transcripts and proteins modulated by K-ras oncogene in the lung tissues of K-ras transgenic mice by omics approaches. Int J Oncol 2009, 34:161-172.

26. Dejean L, Beauvoit B, Bunoust O, Guerin B, Rigoulet M: Activation of Ras cascade increases the mitochondrial enzyme content of respiratory competent yeast. Biochem Biophys Res Commun 2002, 293:1383-1388.

27. Kinkade CW, Castillo-Martin M, Puzio-Kuter A, Yan J, Foster TH, Gao H, Sun Y, Ouyang X, Gerald WL, Cordon-Cardo C, Abate-Shen C: Targeting AKT/mTOR and ERK MAPK signalling inhibits hormone-refractory prostate cancer in a preclinical mouse model. J Clin Invest 2008, 118:3051-3064.

28. Horimoto M, Resnick MB, Konkin TA, Routhier J, Wands JR, Baffy G: Expression of uncoupling protein-2 in human colon cancer. Clin Cancer Res 2004, 10:6203-6207.

29. Lorenzo M, Valverde AM, Teruel T, Alvarez A, Benito M: p21 ras induced differentiation-related gene expression in fetal brown adipocyte primary cells and cell lines. Cell Growth Differ 1996, 7:1251-1259.

30. Chen Y, Cairns R, Papandreou I, Koong A, Denko NC: Oxygen consumption can regulate the growth of tumors, a new perspective on the Warburg effect. PLoS One 2009, 4:e7033.

31. Taetle R, Rosen F, Abramson I, Venditti J, Howell S: Use of nude mouse xenografts as preclinical drug screens: in vivo activity of established chemotherapeutic agents against melanoma and ovarian carcinoma xenografts. Cancer Treat Rep 1987, 71:297-304.

doi:10.1186/1476-4598-11-60

Cite this article as: Telang et al:: Cytochrome coxidase is activated by the oncoprotein Ras and is required for A549 lung adenocarcinoma growth. Molecular Cancer 2012 11:60.

\section{Submit your next manuscript to BioMed Central and take full advantage of:}

- Convenient online submission

- Thorough peer review

- No space constraints or color figure charges

- Immediate publication on acceptance

- Inclusion in PubMed, CAS, Scopus and Google Scholar

- Research which is freely available for redistribution

Submit your manuscript at www.biomedcentral.com/submit
Biomed Central 